\title{
Beneficial Use of Nano-Silica in Concrete: A Review
}

\author{
Aly Said ${ }^{1}$, Achraf Ayad ${ }^{2}$ and Mohamed Zeidan ${ }^{3}$ \\ ${ }^{1}$ Associate Professor, The Pennsylvania State University, USA \\ ${ }^{2}$ Graduate Student, The Pennsylvania State University, USA \\ ${ }^{3}$ Assistant Professor, Southeastern Louisiana University, USA
}

Received: 眥January 28, 2018; Published: 眥 February 05, 2018

*Corresponding author: Aly Said, Architectural Engineering Department, The Pennsylvania State University, 104 Engineering Unit A, University Park, PA 16802,USA

\section{Abstract}

Nano-silica and its use in cement-based materials, especially concrete, has been the focus of many scientific studies. This is because cement production is an energy-intensive process and because concrete is the most used construction material worldwide. Accordingly, it is very important to understand the complexity of concrete, improve its performance and durability. This paper reviews the existing literature on the effects of nano-silica on concrete mechanical properties, microstructure and cement hydration.

Abbreviations: NS: Nano-Silica; SEM: Scanning Electron Microscopy; XRD: X-ray diffraction; FTIR: Fourier transformation infrared spectroscopy; TGA: Thermogravimetric analysis; ASR: Alkali-Silica Reaction

\section{Introduction}

The use of nanomaterials in construction is progressively gaining popularity for the last few decades since they show significant enhancements in materials' performance. Recent studies helped to improve our understanding of nano-silica's role in the cementitious matrix. This includes cement hydration, mechanical properties and microstructure of concrete. Some of these effects are still not fully understood however. This also involves the characterization of the nano-structure of cementitious matrix in order to evaluate its effects on concrete performance using advanced characterization techniques. Furthermore, the manipulation of the nano-structure of the cementitious matrix can create superior qualities like enhanced mechanical properties, reduced porosity and even self-healing capabilities [1-3]. Further information on the importance and the problems related to the use of nanotechnology in concrete is available in the literature. In recent years, researchers investigated the effects of using nano-silica (NS) in cement pastes [4], mortars [5] and concrete [6]. They found that using small dosages of NS improves the early age and the 28-day strength gain which is attributed to the accelerating effect, pozzolanic reaction, reduced porosity and enhanced interfacial transition zone [7-9].

\section{Effect on Mechanical Properties and Porosity}

The mechanical properties of cement mortars containing nano particles specifically nano-SiO2 and nano- $\mathrm{FeO} 3$ were studied by
Hui Li et al. [10] They observed that mortars with nano-SiO2 and nano-FeO3 achieved higher compressive and flexural strength as compared to the reference samples. Scanning Electron Microscopy (SEM) imaging revealed that nano particles did not only act as fillers but also improved the microstructure of the cement paste and promoted cement hydration. Supit, Shaikh [11] examined the durability of high-volume fly ash concrete incorporating NS. They concluded that using NS improved the compressive strength even at small replacement ratios. Moreover, the study showed that replacing $2 \%$ and $4 \%$ of cement by NS resulted in similar performance. The porosity and chloride permeability were significantly reduced after using $2 \%$ NS as compared to when only $15-20 \%$ fly ash was used. NS was also found to improve, even at small replacements, the interfacial transition zone along with the shrinkage performance.

Said et al. [12,13] found that using small dosages of NS in concrete enhanced the mechanical properties and the durability of concrete. In mixtures with $30 \%$ of cement replaced by fly ash and 3-6\% NS, concrete achieved better compressive strength as compared to the mixtures without NS. Similarly, a $14 \%$ and a $32 \%$ increase in the tensile strength were observed in the mixtures when 3\% and 6\% NS were used, respectively. Rapid chloride ion penetration test and mercury intrusion porosimetry were used to assess the porosity. Results indicated that the addition of nanosilica significantly improves in the values of the passing charges 
in chloride ion penetration resistivity. Adding NS also reduced the porosity and refined the pore structure.

\section{Effect on Cement Hydration}

The characteristics of the hydration of cement mortars with NS particles were also investigated by Jo et al. [14]. Scanning electron microscopy was used to monitor the hydration and it was indicated that the addition of NS leads to improvements in the microstructure of the pastes. Mixtures with NS showed a denser, more compact microstructure as compared to Portland cement pastes that had needle-shaped hydrates which reflects the presence of calcium hydroxide. Based on these results, NS not only behaves as filler but also promotes the pozzolanic reaction.

A study by Singh et al. [15] investigated the early hydration of tricalcium silicate in the presence of NS particles. They used X-ray diffraction (XRD), Fourier transformation infrared spectroscopy (FTIR), SEM and Thermogravimetric analysis (TGA) to qualitatively and quantitatively evaluate the additional C-S-H formed. XRD results showed an increase in the intensity of C-S-H peak in the presence of NS indicating a formation of a secondary C-S-H at the early stage of hydration. A gradual decrease in the 850-950 cm-1 peak was monitored to indicate the consumption of $\mathrm{C} 3 \mathrm{~S}$ with respect to time. Their results showed that the addition of nano-silica caused an additional C-S-H formation ( $\sim 50 \%$ more), accelerated the hydration and polymerization process and created a denser C-S-H.

Björnström et al. [16] studied the accelerating effects of NS on the formation of $\mathrm{C}-\mathrm{S}-\mathrm{H}$. The diffuse reflectance FTIR spectra showed that adding NS to cement paste increased both of the consumption of $\mathrm{C} 3 \mathrm{~S}$ and the formation of C-S-H. It was found that increasing the colloidal silica from $1 \%$ to $5 \%$ by weight produced a higher acceleration effect even though smaller dosages were still considerably effective. The main conclusion was that the accelerating effects take place primarily during the initial hydration (4 - 12 hours) and that the acceleration is due to the nucleation effect of the NS particles due to their large surface area.

\section{Effect on Durability}

The use of NS to mitigate the alkali-silica reaction (ASR) in concrete was investigated by $[17,18]$. The study used the accelerated mortar bar testing to evaluate the performance of NS as well as its combination with fly ash and metakaolins in mitigating ASR. The study showed that NS is more effective mitigating ASR when used in combination with pozzolans such as fly ash and metakaolins. The authors attributed this to the fact that NS accelerates pozzolanic reaction, thus limiting the amount of calcium hydroxide required for ASR.

Zeidan et al. [18] investigated the effect of NS on physical salt attack. This phenomenon, which is also referred to as salt weathering, occurs in concrete in contact with soils containing high concentration of salts which gets absorbed into concrete with ground water through capillary action. Subsequently, water evaporates at the surface increasing the concentration of salts in the pores causing efflorescence and surface scaling. The study showed that adding NS to Portland cement enhanced the resistance of concrete to PSA, yet this enhancement decreased for concrete containing fly ash.

\section{Conclusion}

The use of NS is a promising approach for reducing the use of Portland cement which can help reduce $\mathrm{CO} 2$ footprint of construction. It was repeatedly shown that NS can enhance the properties of cement-based materials from many aspects. NS can enhance the mechanical properties of concrete, accelerate cement hydration by means of nucleation effect, and reduce porosity of cement paste through the filler action. However, further research is needed to enhance our understanding of mechanisms of concrete properties enhancements involving NS, especially in the presence of supplementary cementitious materials. Further research is needed to assess NS agglomeration and its effect on workability and means of improving its economy for widespread implementation.

\section{References}

1. Scrivener KL, Kirkpatrick RJ (2008) Innovation in use and research on cementitious material. Cem Concr Res 38 (2): 128-136.

2. Sanchez F, Zhang L, Ince C (2009) Multi scale performance and durability of carbon nanofiber cement composites. In Bittnar Z, Bartos PJM, Nemecek J, Smilauer V, Zeman J, (eds). Nanotechnology in construction: proceedings of the $\mathrm{NICOM}_{3}\left(3^{\text {rd }}\right.$ international symposium on nanotechnology in construction) Prague, Czech Republic 345-350.

3. Jennings HM, Bullard JW, Thomas JJ, Andrade JE, Chen JJ, et al. (2008) Characterization and modeling of pores and surfaces in cement paste: correlations to processing and properties. J Adv Concr Technol 6(1): 5-29.

4. Qing Ye, Zhang Zhenan, Kong Devu,heng Rohnsheng (2007) Influence of nano- $\mathrm{SiO}_{2}$ addition on properties of hardened cement paste as compared with silica fume. Construction and building materials 21(3): 539-545.

5. Li Hui, Hui gang Xiao, Jie Yuan, Jing ou (2004) Microstructure of cement mortar with nano particles. Composites Part B Engineering 35(2): 185189.

6. Schoepfer J, A Maji (2009) An investigation into the effect of silicon dioxide particle size on the strength of concrete. Special Publication 267: 45-58.

7. Sobolev K, Ferrada Gutierrez M (2005) How nanotechnology can change the concrete world: part 2. Am Ceram Soc Bull 84 (11): 16-19.

8. Sobolev K, Shah SP (2008) SP 254 Nanotechnology of concrete: recent developments and future perspectives. Detroit: American Concrete Institute

9. Bittnar Z, Bartos PJM, Nemecek J, Smilauer V, Zeman J, (2009) (eds) Nanotechnology in construction: proceedings of the NICOM3 (3rd international symposium on nanotechnology in construction). Prague Czech Republic Springer Verlag Berlin Heidelberg 438.

10. Supit, Steve Wilben Macquarie, Faiz Uddin Ahmed Shaikh (2015) Durability properties of high volume fly ash concrete containing nano silica. Materials and structures 48(8): 2431-2445.

11. Said, Aly M, Mohamed S Zeidan (2009) Enhancing the reactivity of normal and fly ash concrete using colloidal nano silica. Special Publication 267: 75-86. 
12. Said A M , Zeidan M S, Bassuoni M T ,Tian Y (2012) Properties of concrete incorporating nano silica. Construction and Building Materials (36): 838-844.

13. Jo Byung Wan,Chang Hyun Kim, Ghi Ho Tae, Jong Bin Park (2007) Characteristics of cement mortar with nano SiO 2 particles. Construction and building materials 21(6 ):1351-1355.

14. Singh Lok P, Anjali Goel, Sriman K, usha Sharma, Geethika Mishra (2015) Hydration studies of cementitious material using silica nanoparticles. Journal of Advanced Concrete Technology 13(7): 345-354.

15. Björnström Joakim, Martinelli, A Matic, L Borejanson, I panas (2004) Accelerating effects of colloidal nano silica for beneficial calcium silicate hydrate formation in cement. Chemical Physics Letters 392(1): 242-248.

16.Zeidan M, Said A (2015) AlkaliSilica Reaction Mitigation Using Nanosilica and Fly Ash. In Nanotechnology in Construction 459-464.

17. Zeidan M, Said A M (2017) Effect of colloidal nano silica on alkali silica mitigation. Journal of Sustainable Cement Based Materials 6(2): 126138.

18. Zeidan M , Bassuoni M T Said A (2017) Physical salt attack on concrete incorporating nano-silica. Journal of Sustainable Cement-Based Materials 6(3): 195-216.

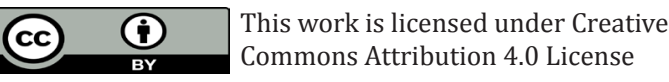

To Submit Your Article Click Here: Submit Article

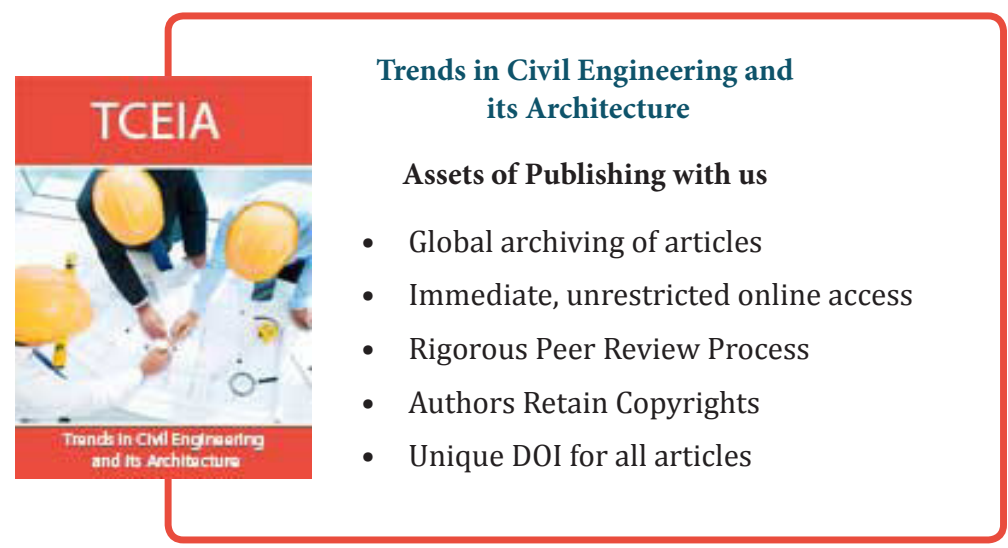

